\title{
Reduced lung dose during radiotherapy for thoracic esophageal carcinoma: VMAT combined with active breathing control for moderate DIBH
}

\author{
Guanzhong Gong, Ruozheng Wang, Yujie Guo, Deyin Zhai, Tonghai Liu, Jie Lu, Jinhu Chen, Chengxin Liu \\ and Yong Yin ${ }^{*}$
}

\begin{abstract}
Background: Lung radiation injury is a critical complication of radiotherapy (RT) for thoracic esophageal carcinoma (EC). Therefore, the goal of this study was to investigate the feasibility and dosimetric effects of reducing the lung tissue irradiation dose during RT for thoracic EC by applying volumetric modulated arc radiotherapy (VMAT) combined with active breathing control (ABC) for moderate deep inspiration breath-hold (mDIBH).

Methods: Fifteen patients with thoracic EC were randomly selected to undergo two series of computed tomography (CT) simulation scans with $\mathrm{ABC}$ used to achieve $\mathrm{mDIBH}$ (representing $80 \%$ of peak DIBH value) versus free breathing (FB). Gross tumor volumes were contoured on different $C T$ images, and planning target volumes (PTVs) were obtained using different margins. For PTV ${ }_{-F B}$, intensity-modulated radiotherapy (IMRT) was designed with seven fields, and VMAT included two whole arcs. For PTV-DIBH, VMAT with three $135^{\circ}$ arcs was applied, and the corresponding plans were named: $I M R T_{-F B}, V M A T_{-F B}$, and VMAT $T_{-D I B H}$, respectively. Dosimetric differences between the different plans were compared.

Results: The heart volumes decreased by $19.85 \%$, while total lung volume increased by $52.54 \%$ in $\mathrm{mDIBH}$, compared to FB $(p<0.05)$. The mean conformality index values and homogeneity index values for VMAT-DIBH $(0.86,1.07)$ were slightly worse than those for $\operatorname{IMRT}_{-F B}(0.90,1.05)$ and VMAT $T_{\text {FB }}(0.90,1.06)(p>0.05)$. Furthermore, compared to IMRT $T_{-F B}$ and VMAT $T_{-F B}$, VMAT respectively $(p<0.05)$; moreover, the $V_{5}, V_{10}, V_{20}$, and $V_{30}$ values for IMRT and $10.65 \%(p>0.05), 12.5 \%$ and $20 \%(p<0.05), 30.77 \%$ and $33.33 \%(p<0.05)$, and $50.33 \%$ and $49.15 \%(p<0.05)$, respectively. However, the heart dose-volume indices were similar between VMAT-DIBH and VMAT FB $_{\text {Which were }}$ lower than IMRT

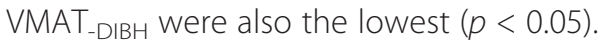

Conclusions: VMAT combined with $A B C$ to achieve $\mathrm{mDIBH}$ is a feasible approach for RT of thoracic EC.

Furthermore, this method has the potential to effectively reduce lung dose in a shorter treatment time and with better targeting accuracy.

Keywords: Esophageal carcinoma, Radiotherapy, Active breathing control, Volumetric modulated arc radiotherapy

\section{Introduction}

Esophageal carcinoma (EC) is one of the most common malignant tumor types worldwide, and its incidence continues to rise [1]. Currently, complete surgical resection is the standard treatment for patients with EC who are medically fit [2]. However, chemo-radiotherapy has also

\footnotetext{
* Correspondence: yongyinsd@163.com

* Correspondence: yongyinsd@163.com
Department of Radiation Oncology, Shandong Cancer Hospital and Institute, Jinan 250117, China
}

achieved promising clinical outcomes for patients with advanced local EC, including squamous cell cancer and adenocarcinoma, as well as for those who are not fit for surgery [2-4]. Radiation therapy (RT) also continues to be a critical component for multimodality systemic treatment of EC [5,6]. However, treatment-related pneumonitis is an acute and toxic side effect that can occur with RT for thoracic EC [7]. Advances have been made in RT technology that can be applied to EC, including the progression 
from two-dimensional RT to three-dimensional conformal radiotherapy (3D-CRT), intensity modulated radiotherapy (IMRT), intensity proton beam radiotherapy (IMPT), volumetric modulated radiotherapy (VMAT), and helical tomotherapy (HT) [5,8-13]. In particular, IMRT, VMAT, and HT have achieved a more conformal and homogeneous dose distribution with the application of beam intensity modulated technology and a multileaf collimator (MLC) system compared with 3D-CRT. VMAT also represents an extension of IMRT which facilitates synchronized variations in gantry speed, dose rate, and the shape of the MLC and jaws [14,15]. Consequently, VMAT can achieve similar, if not better, dose distribution compared with IMRT with shorter treatment times and fewer monitor units (MUs) [15-19]. The application of VMAT to EC RT has obtained similar results, although a more extensive low dose region is involved which can affect normal tissue [9-13]. However, given the shorter duration of the treatment associated with VMAT and the other technologies available, the damage to normal tissue can be minimized [19].

During RT for thoracic EC, displacement and deformation induced by breath motion are critical factors which affect treatment accuracy and precision. For example, in a report by Yanashita et al. [20], the average displacements in the cranial-caudal $(\mathrm{CC})$ for the upper, middle, and lower thoracic esophagus were $3.2 \mathrm{~mm}$ (range, $0.7-5.5 \mathrm{~mm}$ ), $6.4 \mathrm{~mm}$ (range, $1.5-14.5 \mathrm{~mm}$ ), and $10.3 \mathrm{~mm}$ (range, $4.0-16.3 \mathrm{~mm}$ ), respectively. In comparison, the displacement of the left-right (L-R) and anterior-posterior (AP) regions were smaller. However, application of active breathing control $(\mathrm{ABC})$ has been observed to reduce the motion induced by respiration [21,22]. In addition, lung volume can be effectively increased with DIBH, and this has the benefit of sparing lung tissue during $\mathrm{RT}$. Therefore, the feasibility and dosimetric features of VMAT combined with $\mathrm{ABC}$ for $\mathrm{mDIBH}$ for RT of thoracic EC were investigated.

\section{Methods}

\section{Patients}

A total of 15 patients ( 5 females, 10 males) ranging in age from $42-65$ y (median, 53.5 y) had pathologically proven thoracic squamous cell EC and were randomly selected from the patients treated at our hospital between March 2010 and December 2011. Cardiopulmonary function for each patient was carefully and objectively examined prior to enrollment in the study to ensure that the patients could coordinate $\mathrm{ABC}$. All of the enrolled patients had good cardiopulmonary function with Karnofsky performance scores (KPS) ranging from 80-90. None of the patients had communication barriers with the administrators of this study, and the breath holding time in $\mathrm{mDIBH}$ with $A B C$ for all patients reached $30 \mathrm{~s}$. This study was approved by the Research Ethics Board of the Shandong Cancer Hospital, and all patients agreed to the conditions of this trial. Completed informed consent forms were obtained from each patient.

\section{Computed tomography (CT) simulation}

For each patient, two serious CT scans were performed using a Philips Brilliance CT Big Bore (Phillips Medical Systems, 96 Highland Heights, OH, USA). For mDIBH, $\mathrm{CT}$ scans were performed using the Elekta $\mathrm{ABC}$ system (Synergy $102^{\mathrm{m}}$, Elekta, Crawley, UK), with the trigger threshold set at $80 \%$ of the peak value of DIBH. In addition, the latter scans were acquired after scanning with FB. Furthermore, breath training was performed at least twice by each patient prior to scanning. Patients were also immobilized using a body vacuum pillow and their arms were extended above their head.

All CT scans were acquired in spiral model (pitch $=0.938$, table speed $=30 \mathrm{~mm} / \mathrm{s}$, reconstruction slice thickness $=$ $3.0 \mathrm{~mm}$ ) and the region scanned extended from the cricothyroid membrane to the lower edge of the liver. All CT images were transmitted to a Varian Eclipse treatment planning system (TPS) (version 8.6.15, Varian Medical Systems, Palo Alto, CA, USA) to determine target volumes, organs at risk (OARs) contouring, and the design of RT plans.

\section{Gross tumor volume (GTV) and planning target volume (PTV) acquisition}

An experienced radiation oncologist contoured the GTV delineated on each CT image, and this region included the tumor and pathologically involved lymph nodes. The clinical target volume (CTV) included the GTV as well as an additional 3-5 cm in the cranio-caudal direction along the esophagus in order to include the possibility of microscopic spread. The PTV for FB plans (PTV $\mathrm{PBB}_{-\mathrm{B}}$ ) included the addition of a $1.5 \mathrm{~cm}$ margin (for internal motion and setup error) which was applied in all three dimensions to the CTV [21]. Furthermore, the PTV for the mDIBH plans ( $\mathrm{PTV}_{\text {-DIBH }}$ ) included an additional $1 \mathrm{~cm}$ margin to account for setup variation and $A B C$ reproducibility (i.e., uncertainty of organ position with $\mathrm{ABC}$ ), in as much as the tumor was immobilized during breath-hold [21-23]. Healthy lung tissue was also included in the total lung volume, while GTV was excluded.

\section{Radiotherapy plans}

Both IMRT and VMAT plans were designed for PTV $\mathrm{P}_{-\mathrm{FB}}$, while VMAT was designed only for PTV -DIBH. $_{\text {. These plans }}$ were administered using a VARIAN Trilogy linear accelerator (Varian Medical Systems, Palo Alto, CA, USA), and were named $\mathrm{IMRT}_{-\mathrm{FB}}, \mathrm{VMAT}_{-\mathrm{FB}}$, and $\mathrm{VMAT}_{-\mathrm{DIBH}}$, respectively. The details of each plan are as follows: 


\section{IMRT $T_{-F B}$ plan}

A step-and-shoot mode was applied for seven coplanar fields with gantry angles set at $0^{\circ}, 51^{\circ}, 102^{\circ}, 153^{\circ}, 204^{\circ}$, $255^{\circ}$, and $306^{\circ}$. The intensity level was 20 and the dose rate was $400 \mathrm{MU} / \mathrm{min}$.

\section{$V M A T_{-F B}$ plan}

Dual coplanar whole arcs were employed. One arc was performed in a clockwise direction from $181^{\circ}$ to $179^{\circ}$ with a $45^{\circ}$ collimator angle. Conversely, the second arc was performed in a counterclockwise direction from $179^{\circ}$ to $181^{\circ}$ with a $315^{\circ}$ collimator angle. The two arcs were optimized simultaneously and were delivered in opposite rotation with a maximum dose rate of $600 \mathrm{MU} / \mathrm{min}$.

\section{VMAT-DIBH plan}

Three partial $135^{\circ}$ arcs were employed according to the breath holding time with $\mathrm{mDIBH}$. The gantry angles of the three arcs were set to start counterclockwise from $179^{\circ}$ to $44^{\circ}$, from $67^{\circ}$ to $292^{\circ}$, and from $316^{\circ}$ to $181^{\circ}$. Moreover, two arcs were allowed to overlap in the mediastinal region. The collimator angle was set to $45^{\circ}$ for the first and third arcs, and at $315^{\circ}$ for the second arc. The maximum dose rate was $600 \mathrm{MU} / \mathrm{min}$.

All plans were optimized using a six megavolts (MV) $\mathrm{X}$-ray, and the objective dose-volume parameters were identical at the beginning of optimization for the different plans, and the parameters were adjusted following optimization until the results approached an ideal setting.

A VARIAN Triliogy linear accelerator equipped with a Millenni-um-120 MLC (120 leaves with a resolution at isocenter of $5 \mathrm{~mm}$ for the inner $20 \mathrm{~cm}$ and $10 \mathrm{~mm}$ for the outer $2 \times 10 \mathrm{~cm}$ ) was used. The gantry speed was set at $4.8^{\circ}$ s. For all plans, the prescription dose for the PTV was 2 Gy/fraction $\times 30$ fractions [24]. Dose constraints for complications included minimizing doses for health lung (named total lung), heart, and spinal cord, while maintaining optimal target coverage and dose uniformity to the target volumes. In addition, the mean PTV dose was normalized to $100 \%$ of the iso-dose line. All dose distributions were computed using an analytical anisotropic algorithm available in the Eclipse planning system, and the dose grid resolution was $2 \mathrm{~mm}$.

\section{Plan evaluations}

The irradiation doses needed to target 1\% and 99\% of the PTVs ( $D_{1 \%}$ and $D_{99 \%}$, respectively) were designated as the maximum and minimum doses, respectively.

Homogeneity index (HI) values were calculated based on the radiation dose ratio of $5 \%$ and $95 \%$ of the PTVs ( $\mathrm{D}_{5 \%}$ and $\mathrm{D}_{95 \%}$, respectively), e.g., $\mathrm{HI}=\mathrm{D}_{5 \%} / \mathrm{D}_{95 \%}$.

Conformality index $(\mathrm{CI})$ values were calculated according to the Van't Riet definition: $\mathrm{CI}=\left(\mathrm{TV}_{\mathrm{RI}} / \mathrm{TV}\right) \times\left(\mathrm{TV}_{\mathrm{RI}} / \mathrm{V}_{\mathrm{RI}}\right)$, where $T V_{R I}$ is the target volume covered by the reference isodose, $\mathrm{TV}$ is the target volume, and $\mathrm{V}_{\mathrm{RI}}$ is the volume of the reference isodose [25]. CI and HI values are ideal when they approach a value of 1 .

Comparisons were made between the different treatment plans in regard to mean irradiation dose, the percentage of the $x$ Gy radiation dose applied to a volume of healthy tissue $\left(\mathrm{V}_{\mathrm{xGy}}\right)$, including for the lung (e.g., $\mathrm{V}_{5}, \mathrm{~V}_{10}, \mathrm{~V}_{20}, \mathrm{~V}_{30}$, and $V_{40}$ ) and heart (e.g., $V_{20}, V_{30}$, and $V_{40}$ ), the maximum radiation dose applied to the spinal cord, MUs, and treatment time.

\section{Statistical analysis}

All results are reported as the average \pm standard deviation $(\bar{X} \pm S)$ and SPSS 16.0 software (IBM, Chicago, IL, USA) was used for all statistical analyses. A paired $t$-test was used to compare total lung and heart volumes, GTV, and PTV for each breath status. One-way analysis of variance (ANOVA) was used to compare indices among different plans, and the difference between two plans was compared using Bonferroni multiple comparison test. Differences were considered statistically significant when the $p$-value was less than 0.05 .

\section{Results}

Comparison of GTV, PTV, total lung and heart volumes

As shown in Table 1, the mean GTV volume determined with $\mathrm{FB}$ was $11.75 \%$, and this was greater than that determined with $\mathrm{mDIBH}$. However, this difference was not statistically significant $(p>0.05)$. In contrast, mean PTV and heart volume decreased $(31.61 \%$ and $19.85 \%$, respectively), while mean total lung volume increased (52.54\%), with $\mathrm{mDIBH}$. Furthermore, each of those differences were statistically significant $(p<0.05)$.

\section{Dose distribution of PTV}

The $\mathrm{D}_{1 \%}$ and $\mathrm{D}_{99 \%}$ were similar among the IMRT $_{-\mathrm{FB}}$, VMAT $_{- \text {FB }}$ and VMAT VIBH $_{\text {-DIans }}(p>0.05)$. The CI and HI values for VMAT $_{\text {-DIBH }}$ were slightly worse than those for IMRT $\mathrm{I}_{-\mathrm{FB}}$ and $\mathrm{VMAT}_{-\mathrm{FB}}$, and these differences were not statistically significant $(p>0.05)$. Meanwhile, $\mathrm{CI}$ and $\mathrm{HI}$ values were similar for the $\mathrm{IMRT}_{-\mathrm{FB}}$ and VMAT $_{\text {-FB }}$ plans $(p>0.05)$.

Table 1 Target volumes and OAR volumes for FB and DIBH CT techniques $\left(\mathrm{cm}^{3}\right)$

\begin{tabular}{lllll}
\hline Volume & FB $\left(\mathbf{c m}^{\mathbf{3}}\right)$ & DIBH $\left(\mathbf{c m}^{\mathbf{3}}\right)$ & $\boldsymbol{T}$ & $\boldsymbol{p}$ \\
\hline GTV & $52.58 \pm 19.72$ & $46.40 \pm 20.78$ & 0.610 & 0.552 \\
PTV & $396.11 \pm 72.43$ & $270.91 \pm 87.02$ & 2.259 & 0.040 \\
Total lung & $4039.40 \pm 79.86$ & $6161.60 \pm 80.85$ & -5.282 & 0.000 \\
Heart & $621.08 \pm 66.84$ & $497.80 \pm 86.58$ & 3.188 & 0.007 \\
\hline
\end{tabular}

GTV Gross tumor volume, PTV Planning target volume. 


\section{Organs at risk (OARs)}

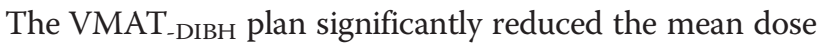
and the $V_{5}$ to $V_{40}$ values for total lung compared with the IMRT $_{\text {-FB }}$ and VMAT $_{\text {-FB }}$ plans (except for $\mathrm{V}_{5}$, as shown in Table 2) $(p<0.05)$. The $\mathrm{V}_{10}$ of $\mathrm{VMAT}_{-\mathrm{FB}}$ was also higher than that of the IMRT $_{\text {-FB }}$ plan, yet the difference was not significant $(p>0.05)$. The mean dose, $V_{5}, V_{20}, V_{30}$, and $\mathrm{V}_{40}$ were also similar for the $\mathrm{IMRT}_{-\mathrm{FB}}$ and $\mathrm{VMAT}_{-\mathrm{FB}}$ plans $(p>0.05)$.

For the heart, the $\mathrm{V}_{20}, \mathrm{~V}_{30}$, and $\mathrm{V}_{40}$ values were similar between the $\mathrm{VMAT}_{\text {-DIBH }}$ and $\mathrm{VMAT}_{\text {-FB }}$ plans $(p>0.05)$, and these values were lower than those of the IMRT $\mathrm{IBB}_{-\mathrm{B}}$ plan. The mean irradiation dose for the heart was also similar between the IMRT $_{-\mathrm{FB}}$ and $\mathrm{VMAT}_{\text {-FB }}$ plans, and these doses were higher than that for the $\mathrm{VMAT}_{\text {-DIBH }}$ plan.

There were no significant differences in the $\mathrm{D}_{\max }$ values for the spinal cord among the plans $(p>0.05)$.

\section{MUs and delivery time}

MUs for the IMRT -FB $_{\text {- }}$ plan $(798.38 \pm 112.99)$ were significantly greater than those for the $\mathrm{VMAT}_{-\mathrm{FB}}(508.25 \pm$ $64.95)$ and VMAT $_{\text {-DIBH }}(506.88 \pm 78.87)$ plans $(p<0.05)$.

Table 2 Dose-volume indices of the PTV and OARs

\begin{tabular}{|c|c|c|c|c|c|}
\hline Variable & IMRT $_{-F B}$ & VMAT $_{-F B}$ & VMAT-DIBH $_{\text {- }}$ & $F$ & $p$ \\
\hline \multicolumn{6}{|l|}{ PTV } \\
\hline$D_{1 \%}(G y)$ & $65.67 \pm 0.41$ & $65.74 \pm 0.35$ & $66.10 \pm 0.44$ & 2.599 & 0.098 \\
\hline$D_{99 \%}(G y)$ & $58.82 \pm 0.52$ & $58.76 \pm 0.51$ & $58.35 \pm 0.41$ & 2.156 & 0.141 \\
\hline $\mathrm{Cl}$ & $0.90 \pm 0.03$ & $0.90 \pm 0.03$ & $0.86 \pm 0.04$ & 2.864 & 0.079 \\
\hline $\mathrm{HI}$ & $1.05 \pm 0.02$ & $1.06 \pm 0.02$ & $1.07 \pm 0.03$ & 2.485 & 0.107 \\
\hline \multicolumn{6}{|l|}{ Total lung } \\
\hline$V_{5}(\%)$ & $83 \pm 9$ & $83 \pm 9$ & $74 \pm 10$ & 2.274 & 0.128 \\
\hline$V_{10}(\%)$ & $64 \pm 9$ & $70 \pm 10$ & $56 \pm 10$ & 4.808 & 0.019 \\
\hline$V_{20}(\%)$ & 26. \pm 5 & $24 \pm 6$ & $18 \pm 4$ & 5.518 & 0.012 \\
\hline$V_{30}(\%)$ & $8 \pm 4$ & $8 \pm 3$ & $4 \pm 2$ & 4.062 & 0.032 \\
\hline$V_{40}(\%)$ & $4 \pm 2$ & $4 \pm 1$ & $2 \pm 0$ & 3.790 & 0.039 \\
\hline$D_{\text {mean }}(G y)$ & $15.45 \pm 2.24$ & $15.30 \pm 2.19$ & $12.57 \pm 1.78$ & 4.884 & 0.018 \\
\hline \multicolumn{6}{|l|}{ Heart } \\
\hline$V_{20}(\%)$ & $71 \pm 2.46$ & $61 \pm 2.77$ & $61 \pm 2.73$ & 0.336 & 0.719 \\
\hline$V_{30}(\%)$ & $38 \pm 2.37$ & $36 \pm 2.35$ & $35 \pm 2.20$ & 0.032 & 0.968 \\
\hline$V_{40}(\%)$ & $22 \pm 1.42$ & $20 \pm 1.40$ & $19 \pm 1.25$ & 0.097 & 0.908 \\
\hline$D_{\text {mean }}$ & $28.83 \pm 8.60$ & $28.82 \pm 8.71$ & $27.32 \pm 8.53$ & 0.080 & 0.923 \\
\hline \multicolumn{6}{|l|}{ Spinal cord } \\
\hline$D_{\max }(G y)$ & $43.54 \pm 2.62$ & $44.83 \pm 2.19$ & $41.25 \pm 4.40$ & 2.542 & 0.103 \\
\hline $\begin{array}{c}\text { Monitor } \\
\text { units }\end{array}$ & $798.38 \pm 11.20$ & $508.25 \pm 6.49$ & $506.88 \pm 7.88$ & 29.156 & 0.000 \\
\hline $\begin{array}{l}\text { Treatment } \\
\text { time }(\mathrm{s})\end{array}$ & $367.64 \pm 3.39$ & $170 \pm 0$ & $124 \pm 0$ & 349.997 & 0.000 \\
\hline
\end{tabular}

$\mathrm{Cl}$ Conformality index, HI Homogeneity index.
The estimated treatment time (from first filed beam-on to the last filed beam-off) for $\mathrm{VMAT}_{\text {-DIBH }}(124 \pm 0 \mathrm{~s})$ was also shorter than that for $\mathrm{VMAT}_{-\mathrm{FB}}(170 \pm 0 \mathrm{~s})$, and was also significantly shorter than the measured treatment time for $\mathrm{IMRT}_{-\mathrm{FB}}(367.64 \pm 33.90 \mathrm{~s})(p<0.05)$.

\section{Discussion}

Previously, VMAT has been shown to achieve better dose delivery with a shorter treatment time and fewer MUs [10-20]. However, VMAT has also been associated with a larger low dose region that affects OARs in some cases [10-17]. Correspondingly, differences in lung $\mathrm{V}_{10}$ between the VMAT $_{\text {-FB }}$ and IMRT $_{\text {-FB }}$ plans in the present study were observed.

Treatment-related pneumonitis represents critical radiation toxicity during RT for thoracic EC, and dose-volume indices are commonly used to predict this side effect [26-28]. While $\mathrm{V}_{20}, \mathrm{~V}_{30}$, and the mean lung dose have been considered classic predictors of treatment-related pneumonitis, the predictive value of $\mathrm{V}_{5}, \mathrm{~V}_{10}$, and $\mathrm{V}_{13}$ have also been considered [29-31]. Therefore, the approach which reduces lung dose for all dose-volume indices, provides sufficient PTV coverage, and achieves perfect dose distribution, is preferred [32]. Accordingly, VMAT represents a preferred method based on its shorter treatment time, fewer MUs, and better dose delivery. Furthermore, in association with $\mathrm{ABC}$, VMAT may potentially spare a greater region of normal tissue from damage [33].

The displacement and deformation of thoracic EC caused by breath motion can significantly affect the precision of RT. In addition, the randomness of breath phase at the scanning moment with $\mathrm{FB}$ can potentially introduce more uncertainty. This is usually compensated for with larger margins. However, if the margins are extended too far, this can increase the probability that radiation injury will occur. Accordingly, the ability to reduce breath motion is important, especially for patients with distal EC. The application of $\mathrm{ABC}$ with $\mathrm{mDIBH}$ was found to further reduce lung radiation dose by increasing the absolute lung volume.

At the beginning of this study, IMRT and ABC were combined for RT of EC. However, the feasibility of this approach was limited by the longer time needed for each field and fraction of IMRT. Each IMRT plan has seven fields, and sometimes one field may be divided into more than one subfield, potentially leading to 14 or more subfields. In addition, patients need to rest for at least six intervals, and this may compromise the reproducibility of the breath hold motion due to muscle fatigue. Second, if one field or subfield is not completed within the breathholding time of the patient, it is difficult to ensure that the relative position of the MLC and PTV will be identical to the moment of beam off. Regarding the VMAT plans, completing a whole arc using segments was not feasible, 
and this approach would waste time and increase uncertainty [33]. Therefore, duration of treatment for each field and fraction, as well as the risk of secondary tumors for patients who survive for an extended period of time, are important factors to consider. In addition, for the whole arc(s) VMAT plans, a larger low dose region affecting OARs was observed, although good dose delivery was achieved in a shorter period of time and with fewer MUs using these plans.

It was observed that the VMAT-DiBH plans with three $135^{\circ}$ arcs were associated with lower CI values than the $\mathrm{IMRT}_{\text {-FB }}$ and $\mathrm{VMAT}_{\text {-FB }}$ plans, although the differences were not significant. A possible reason for this phenomenon is that $\mathrm{VMAT}_{\text {-DIBH }}$ involves fewer control points than the other plans. Thus, another $\operatorname{arc}<135^{\circ}$ may be needed to raise CI values [34]. For the $\mathrm{VMAT}_{-\mathrm{FB}}$ plan, it contained $3-4$ arcs (including 2-3 rest times for the patient), which is reasonable to ensure the reproducibility and stability of the breath hold motion based on our experience with $\mathrm{RT}$ and $\mathrm{ABC}$ applied to breast cancer, lung cancer, and hepatocellular carcinoma.

$A B C$ has been shown to be an effective approach for improving the precise application of RT for thoracic and abdominal cancer. Specifically:

(1) $\mathrm{ABC}$ facilitates the positioning of the target volume of a tumor by limiting the motion of the target volume induced by respiration. Consequently, margins between the CTV and the PTV were able to be reduced by effectively restricting respiratory motion. Moreover, OARs were able to be spared by ensuring accurate positioning of the tumor [33].

(2)DIBH can increase lung volume, thereby sparing normal lung tissue during treatment. Moreover, the use of $A B C$ to improve DIBH has previously been applied to RT for breast cancers, lung cancers, and lymphomas, with positive results reported [35].

(3) The use of $A B C$ to achieve varying degrees of breath status, including DIBH, deep expiratory hold (DEBH), or mDIBH, potentially changes the spatial relationship between tumor and OARs, and this may subsequently affect the dose-volume of OARs.

In this study, the lower radiation dose for lung tissue can be attributed to the reduction in PTV margins and the increase in lung volume with DIBH. Total lung volume and dose-volume parameters for the lung are two important factors [36]. Displacement of the ECs in different directions was not able to be clearly determined in the present study, although it is possible to measure these parameters using 4D-CT. In simulations, respiratory phase randomness was associated with too much uncertainty regarding $\mathrm{GTV}_{\mathrm{FB}}$, and this uncertainty was effectively reduced with the use of DIBH. Furthermore, the $5 \mathrm{~mm}$ reduction in tumor margins was found to be moderate and reasonable in the present study [20,21], and dosimetric benefit was maintained in relation to lung volume if the same margins were applied

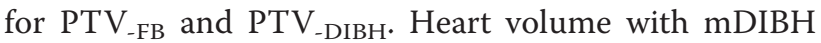
was also significantly smaller than with $\mathrm{FB}$, while the dose-volume indices for $\mathrm{VMAT}_{\text {-DIBH }}$ were lower than

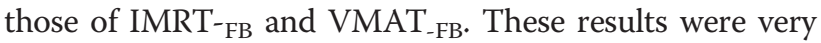
promising, although the dose-volume indices did not differ significantly. This may be due to the spatial limitation of heart beats during DIBH, reductions in tumor margins, and the increased distance for PTV.

Overall, VMAT combined with $\mathrm{ABC}$ in the present study achieved good, accurate dose distribution, involved a shorter treatment time, and reduced low dose exposure of OARs. The total treatment time was approximately $115 \mathrm{~s}$, which includes the rest time for the patient and the gantry rotation time for beam preparation. Moreover, this period of time is shorter than that needed to complete double whole arc VMAT. CI values can also increase with low speed gantry rotation, and this speed should be as fast as possible, as soon as possible, in order to reduce the uncertainty of RT, especially when applying $\mathrm{ABC}$ [37].

The prescription dose used in this study was $60 \mathrm{~Gy} / 30 \mathrm{~F}$, and was selected based on survival rates versus escalating dose. In addition, another radiation therapy oncology group (RTOG) had previously demonstrated that a dose of 50.4 Gy was associated with an acceptable clinical outcome $[38,39]$. In particular, lung irradiation dose was found to be the major limiting factor for increasing the PTV dose. However, using VMAT with ABC for mDIBH, the prescription dose could be higher. Although the dosimetric benefits were evaluated by comparing treatment strategies, the reproducibility of these results need to be confirmed, particularly regarding the clinical benefit of the breath hold motion. However, clinical outcome may not be obtained in a short period of time. Therefore, incorporation of image-guided RT could further improve the precision of the currently proposed method without significantly increasing treatment time. In addition, patients treated in the present study will continue to be monitored in order to verify the current results and to improve the design of future studies.

\section{Conclusions}

VMAT combined with $\mathrm{ABC}$ for $\mathrm{mDIBH}$ achieved a similar dose distribution for tumor targets and spared OARs to a greater extent than IMRT and double whole arc VMAT with FB. Therefore, VMAT combined with ABC for $\mathrm{mDIBH}$ is recommended for thoracic EC patients with good cardiopulmonary function.

\section{Competing interests}

The authors declare that they have no competing interests. 


\section{Authors' contributions}

All authors read and approved the final manuscript. Guanzhong Gong, Ruozheng Wang, Yujie Guo, Deyin Zhai, Tonghai Liu, Jie Lu and Jinhu Chen carried out the design and optimization of IMRT and VMAT treatment plans, and Chengxin Liu carried out the statistics of this study, and Yong Yin administrated the study. All authors participated in the sequence alignment and drafted the manuscript.

\section{Acknowledgements}

This research was supported by the National Natural Science Fund of China (No. 81301936).

Received: 16 July 2013 Accepted: 8 December 2013

Published: 20 December 2013

\section{References}

1. Siegel R, Naishadham D, Jemal A: Cancer statistics, 2012. CA Cancer J Clin 2012, 62:10-29.

2. Shahbaz Sarwar CM, Luketich JD, Landreneau RJ, Abbas G: Esophageal cancer: an update. Int J Surg 2010, 8:417-422.

3. Pantling AZ, Gossage JA, Mamidanna R, Newman G, Robinson A, Manifold DK, Hale PC: Outcomes from chemoradiotherapy for patients with esophageal cancer. Dis Esophagus 2011, 24:172-176.

4. Zhang J, Peng F, Li N, Liu Y, Xu Y, Zhou L, Wang J, Zhu J, Huang M, Gong Y Salvage concurrent radio-chemotherapy for post-operative local recurrence of squamous-cell esophageal cancer. Radiat Oncol 2012, 7:93.

5. Tu L, Sun L, Xu Y, Wang Y, Zhou L, Liu Y, Zhu J, Peng F, Wei Y, Gong Y Paclitaxel and cisplatin combined with intensity-modulated radiotherapy for upper esophageal carcinoma. Radiat Oncol 2013, 8:75.

6. Fakhrian K, Gamisch N, Schuster T, Thamm R, Molls M, Geinitz H: Salvage radiotherapy in patients with recurrent esophageal carcinoma. Strahlenther Onkol 2012, 188:136-142.

7. Wang S, Liao Z, Wei X, Liu HH, Tucker SL, Hu C, Ajani JA, Phan A, Swisher SG, Mohan R, Cox JD, Komaki R: Association between systemic chemotherapy before chemoradiation and increased risk of treatment-related pneumonitis in esophageal cancer patients treated with definitive chemoradiotherapy. J Thorac Oncol 2008, 3:277-282.

8. Mizumoto M, Sugahara S, Nakayama H, Hashii H, Nakahara A, Terashima H, Okumura T, Tsuboi K, Tokuuye K, Sakurai H: Clinical results of proton-beam therapy for locoregionally advanced esophageal cancer. Strahlenther Onkol 2010, 186:482-488

9. Vosmik M, Petera J, Sirak I, Hodek M, Paluska P, Dolezal J, Kopacova M: Technological advances in radiotherapy for esophageal cancer. World J Gastroenterol 2010, 16:5555-5564

10. Benthuysen L, Hales L, Podgorsak MB: Volumetric modulated arc therapy vs. IMRT for the treatment of distal esophageal cancer. Med Dosim 2011 36:404-409.

11. Vivekanandan N, Sriram P, Kumar SA, Bhuvaneswari N, Saranya K: Volumetric modulated arc radiotherapy for esophageal cancer. Med Dosim 2012, 37:108-113.

12. Martin S, Chen JZ, Rashid Dar A, Yartsev S: Dosimetric comparison of helical tomotherapy, RapidArc, and a novel IMRT \& Arc technique for esophageal carcinoma. Radiother Oncol 2011, 101:431-437.

13. Yin Y, Chen J, Xing L, Dong X, Liu T, Lu J, Yu J: Applications of IMAT in cervical esophageal cancer radiotherapy: a comparison with fixed-field IMRT in dosimetry and implementation. J App/ Clin Med Phys 2011 12:48-57.

14. Wang PM, Hsu WC, Chung NN, Chang FL, Fogliata A, Cozzi L: Radiation treatment with volumetric modulated arc therapy of hepatocellular carcinoma patients. Early clinical outcome and toxicity profile from a retrospective analysis of 138 patients. Radiat Oncol 2012, 7:207.

15. Richetti A, Fogliata A, Clivio A, Nicolini G, Pesce G, Salati E, Vanetti E, Cozzi L: Neo-adjuvant chemo-radiation of rectal cancer with volumetric modulated arc therapy: summary of technical and dosimetric features and early clinical experience. Radiat Oncol 2010, 5:14.

16. Pesce GA, Clivio A, Cozzi L, Nicolini G, Richetti A, Salati E, Valli M, Vanetti E, Fogliata A: Early clinical experience of radiotherapy of prostate cancer with volumetric modulated arc therapy. Radiat Oncol 2010, 5:54.

17. Wolff HA, Wagner DM, Christiansen H, Hess CF, Vorwerk H: Single fraction radiosurgery using Rapid Arc for treatment of intracranial targets. Radiat Oncol 2010, 5:77.
18. Scorsetti M, Alongi F, Fogliata A, Pentimalli S, Navarria P, Lobefalo F, Garcia-Etienne C, Clivio A, Cozzi L, Mancosu P, Nicolini G, Vanetti E, Eboli M, Rossetti C, Rubino A, Sagona A, Arcangeli S, Gatzemeier W, Masci G, Torrisi R, Testori A, Alloisio M, Santoro A, Tinterri C: Phase I-Il study of hypofractionated simultaneous integrated boost using volumetric modulated arc therapy for adjuvant radiation therapy in breast cancer patients: a report of feasibility and early toxicity results in the first 50 treatments. Radiat Oncol 2012, 7:145.

19. Sriram P, Syamkumar SA, Kumar JS, Prabakar S, Dhanabalan R, Vivekanandan N: Adaptive volumetric modulated arc treatment planning for esophageal cancers using cone beam computed tomography. Phys Med 2012, 28:327-332.

20. Yamashita H, Kida S, Sakumi A, Haga A, Ito S, Onoe T, Okuma K, Ino K, Akahane M, Ohtomo K, Nakagawa K: Four-dimensional measurement of the displacement of internal fiducial markers during 320-multislice computed tomography scanning of thoracic esophageal cancer. Int J Radiat Oncol Biol Phys 2011, 79:588-595.

21. Lorchel F, Dumas JL, Noël A, Wolf D, Bosset JF, Aletti P: Esophageal cancer: determination of internal target volume for conformal radiotherapy. Radiother Oncol 2006, 80:327-332.

22. Wong JW, Sharpe MB, Jaffray DA, Kini VR, Robertson JM, Stromberg JS, Martinez AA: The use of active breathing control $(A B C)$ to reduce margin for breathing motion. Int J Radiat Oncol Biol Phys 1999, 44:911-919.

23. Patel AA, Wolfgang JA, Niemierko A, Hong TS, Yock T, Choi NC: Implications of respiratory motion as measured by four-dimensional computed tomography for radiation treatment planning of esophageal cancer. Int J Radiat Oncol Biol Phys 2009, 74:290-296.

24. Asakura H, Hashimoto T, Zenda S, Harada H, Hirakawa K, Mizumoto M, Furutani K, Hironaka S, Fuji H, Murayama S, Boku N, Nishimura T: Analysis of dose-volume histogram parameters for radiation pneumonitis after definitive concurrent chemoradiotherapy for esophageal cancer. Radiother Oncol 2010, 95:240-244

25. Cilla S, Macchia G, Digesù C, Deodato F, Romanella M, Ferrandina G, Padula GD, Picardi V, Scambia G, Piermattei A, Morganti AG: 3D-Conformal versus intensity-modulated postoperative radiotherapy of vaginal vault: a dosimetric comparison. Med Dosim 2010, 35:135-142.

26. Blom Goldman U, Wennberg B, Svane G, Bylund H, Lind P: Reduction of radiation pneumonitis by V20-constraints in breast cancer. Radiat Oncol 2010, 5:99.

27. Vogelius IR, Bentzen SM: A literature-based meta-analysis of clinical risk factors for development of radiation induced pneumonitis. Acta Oncol 2012, 51:975-983.

28. Dang J, Li G, Ma L, Diao R, Zang S, Han C, Zhang S, Yao L: Predictors of grade $\geq 2$ and grade $\geq 3$ radiation pneumonitis in patients with locally advanced non-small cell lung cancer treated with three-dimensional conformal radiotherapy. Acta Oncol 2012, 52:1175-1180.

29. Yang GY, McClosky SA, Khushalani NI: Principles of modern radiation techniques for esophageal and gastroesophageal junction cancers. Gastrointest Cancer Res 2009, 3(2 Suppl):S6-S10.

30. Schallenkamp JM, Miller RC, Brinkmann DH, Foote T, Garces YI: Incidence of radiation pneumonitis after thoracic irradiation: dose-volume correlates. Int J Radiat Oncol Biol Phys 2007, 67:410-416.

31. Wang SL, Liao Z, Vaporciyan AA, Tucker SL, Liu H, Wei X, Swisher S, Ajani JA, Cox JD, Komaki R: Investigation of clinical and dosimetric factors associated with postoperative pulmonary complications in esophageal cancer patients treated with concurrent chemoradiotherapy followed by surgery. Int J Radiat Oncol Biol Phys 2006, 64:692-699.

32. Vogelius IS, Westerly DC, Cannon GM, Bentzen SM: Hypofractionation does not increase radiation pneumonitis risk with modern conformal radiation delivery techniques. Acta Oncol 2010, 49:1052-1057.

33. Gong GZ, Yin Y, Xing LG, Guo YJ, Liu T, Chen J, Lu J, Ma C, Sun T, Bai T, Zhang G, Wang R: RapidArc combined with the active breathing coordinator provides an effective and accurate approach for the radiotherapy of hepatocellular carcinoma. Strahlenther Onkol 2012, 188:262-268.

34. Guckenberger M, Richter A, Krieger T, Wilbert J, Baier K, Flentje M: Is a single arc sufficient in volumetric-modulated arc therapy (VMAT) for complex-shaped target volumes? Radiother Oncol 2009, 93:259-265.

35. Paumier A, Ghalibafian M, Gilmore J, Beaudre A, Blanchard P, el Nemr M, Azoury F, al Hamokles H, Lefkopoulos D, Girinsky T: Dosimetric benefits of intensity-modulated radiotherapy combined with the deep-inspiration breath-hold technique in patients with mediastinal Hodgkin's lymphoma. Int J Radiat Oncol Biol Phys 2012, 82:1522-1527. 
36. Liao Z, Cox JD, Komaki R: Radiochemotherapy of esophageal cancer. J Thorac Oncol 2007, 2:553-568.

37. Pasler M, Wirtz H, Lutterbach J: Impact of gantry rotation time on plan quality and dosimetric verification-volumetric modulated arc therapy (VMAT) vs. intensity modulated radiotherapy (IMRT). Strahlenther Onkol 2011, 187:812-819.

38. Wolf M, Zehentmayr F, Niyazi M, Ganswindt U, Haimerl W, Schmidt M, Hölzel D, Belka C: Long-term outcome of mitomycin C- and 5-FU-based primary radiochemotherapy for esophageal cancer. Strahlenther Onkol 2010, 186:374-381

39. Patonay P, Naszály A, Mayer A: Simultaneous radiochemotherapy and endoluminal HDR brachytherapy in esophageal cancer. Strahlenther Onkol 2007, 183:94-98.

doi:10.1186/1748-717X-8-291

Cite this article as: Gong et al:: Reduced lung dose during radiotherapy for thoracic esophageal carcinoma: VMAT combined with active breathing control for moderate DIBH. Radiation Oncology 2013 8:291.

\section{Submit your next manuscript to BioMed Central and take full advantage of:}

- Convenient online submission

- Thorough peer review

- No space constraints or color figure charges

- Immediate publication on acceptance

- Inclusion in PubMed, CAS, Scopus and Google Scholar

- Research which is freely available for redistribution 